\title{
GENDER DETERMINATION FROM FINGERPRINTS
}

\author{
Karki RK ${ }^{1}$, Singh $\mathrm{PK}^{2}$
}

\section{ABSTRACT}

INTRODUCTION: Fingerprints are one of the most mature biometric technologies and are considered legitimate proofs of evidence in courts of law for human identification all over the world. There is relatively less research has been done for its use in this field of gender identification. Hence, the present study was done to look the correlation between fingertip patterns with the gender of an individual.

METHODS: Study was carried out over a period of one month among 200 medical students ( 100 male \& 100 female) belonging to the age group 18-25 years of Kathmandu University School of Medical Sciences, Dhulikhel, Kavre.

RESULTS: Each finger print is unique; loops are the most commonly occurring fingerprint pattern while arches are the least common. Males have a higher incidence of whorls and females have a higher incidence of loops.

CONCLUSION: We can conclude that there is an association between distribution of fingerprint patterns and gender and thus prediction of gender of a person is possible based on his fingerprint pattern.

KEYWORDS: Fingerprints, Gender, Identification

1. Assistant Professor, Department of Forensic Medicine and Toxicology, Kathmandu University School of Medical Sciences, Dhulikhel, Kavre, Nepal

2. Lecturer, Department of Forensic Medicine and Toxicology, Kathmandu University School of Medical Sciences, Dhulikhel, Kavre, Nepal

\author{
For correspondence \\ Dr. Raj Kumar Karki, MBBS, MD \\ Department of Forensic Medicine and Toxicology, \\ Kathmandu University School of Medical Sciences, (KU), \\ Dhulikhel, Kavre, Nepal \\ E-mail:rkkarki17@yahoo.com
}




\section{INTRODUCTION}

Identity is a set of physical characteristics, functional or psychic, normal or pathological that defines an individual. Recently, there has been an increasing interest in biometric technologies for human identification based on one's individual features. The various identification data used are fingerprints, handwriting, bite marks, DNA fingerprinting etc. ${ }^{1}$ Fingerprints are constant and individualistic and form the most reliable criteria for identification., ${ }^{2,3}$ People can be identified by number of features such as face, height, body, shape, gait, voice sex etc. Sex is among the most important information that discriminates individuals. Researchers addressed the use of fingerprint for gender identification which will be more helpful in short listing the suspects. In recent times, more and more civilian and commercial applications are either using or actively considering using fingerprint-based identification because of the availability of inexpensive and compact solid state scanners as well as its superior and proven matching performance over other biometric technologies. Skin on human fingertips contains ridges and valleys which together forms distinctive patterns. These patterns are fully developed during gestational period and are permanent throughout whole lifetime. Prints of those patterns are called fingerprints. Injuries like cuts, burns and bruises can temporarily damage quality of fingerprints but when fully healed, patterns will be restored. Fingerprints are the most effective identification of evidence. Especially in criminal identification, in case of missing person or disaster victims, the fingerprint identification is the first simple individual identification before using complicated technique such as DNA analysis and now it has become more automated due to advancements in the computing capabilities.

\section{MATERIALSAND METHODS}

After obtaining clearance from institutional ethics committee, this prospective cross sectional study was carried out over a period of one month among 200 medical students belonging to the Kathmandu University School of Medical Sciences, Dhulikhel, Kavre. Students with permanent scars on their fingers or thumbs, with any hand deformities due to injury, birth defect or disease, those having worn fingerprints, extra, webbed or bandaged fingers, were excluded from the study. Each subject was asked to wash his hands thoroughly with soap and water and dry them using a towel. Then they were asked to press their fingertip on the stamp pad and then to the paper to transfer the fingerprint impression. The same method was repeated for all the fingers of both hands. In this way, the plain fingerprints of all the ten digits were taken separately on the respective blocks on the same sheet of paper. Care was taken to avoid sliding of fingers to prevent smudging of the print. After the fingerprints were acquired, sex and age were noted. Each subject was assigned a serial number. The fingerprint patterns were studied with the help of a magnifying lens and were identified as: Loops, Whorls and Arches based on the appearance of ridge lines according to Henry's system of classification. This system assigns each finger a number according to the order in which is it located in the hand, beginning with the right thumb as number one and ending with the left little finger as number 10 . The distribution of dermatoglyphic fingertip patterns in both hands of individuals and its relationship with gender was evaluated and analyzed statistically.

\section{RESULTS}

A total of 200 subjects participated in the study out of which 100 were males and 100 were females from 1825 years of age. Fingerprint pattern analysis of 2000 fingers ( 200 subject $\mathrm{X} 10$ fingers of right and left hand) showed that, loops were the most common pattern, followed by whorls while arches were present in a smaller percentage of the study group. (Table 1)

Table 1: General distributions of primary fingerprint patterns in all fingers of both hands

\begin{tabular}{|l|l|}
\hline Pattern of fingerprint & Number (\%) \\
\hline Loops & $1242(62.10)$ \\
\hline Whorls & $591(29.55)$ \\
\hline Arches & $167(8.35)$ \\
\hline Total & $2000(100)$ \\
\hline
\end{tabular}

Frequency of loops was found to be higher in females $(68.60 \%)$ than in males $(35.80 \%)$ whereas whorls were more frequent in males $(57.90 \%)$ as compared to females (27.10\%). (Table 2)

Table 2: Distributions of Pattern of fingerprints among Males and Females

\begin{tabular}{|l|l|l|}
\hline \multicolumn{1}{|c|}{ Type } & \multicolumn{1}{c|}{ Male (\%) } & \multicolumn{1}{c|}{ Female (\%) } \\
\hline Loops & $358(35.80 \%)$ & $686(68.60 \%)$ \\
\hline Whorls & $579(57.90 \%)$ & $271(27.10 \%)$ \\
\hline Arches & $63(6.30 \%)$ & $43(4.30 \%)$ \\
\hline
\end{tabular}


The ridges density of all the finger prints noted of $25 \mathrm{~mm}^{2}$. The result have shown that a fingerprint ridge of $<12$ ridges $/ 25 \mathrm{~mm}^{2}$ is more likely of male origin and finger print ridge of $>14$ ridges $/ 25 \mathrm{~mm}^{2}$ is more likely of female origin. So that female tends to have a significantly higher ridge density than male.

\section{DISCUSSION}

For over centuries, fingerprint has been used for both identification and verification because of its uniqueness. A fingerprint contains three level of information. Level 1 feature contain macro details of fingerprint such as ridge flow and pattern type e.g. arch, loop, whorl etc. Level 2 features refer to the Galton characteristics or minute, such as ridge bifurcation or ridge termination e.g. eye, hook, bifurcation, ending etc. Level 3 features include all dimensional attributes of ridge e.g. ridge path deviation, width, shape, pores, edge contour, ridges breaks, creases, scars and other permanent details. ${ }^{4}$ Within today's environment of increased importance of security and organization, identification andauthentication methods have developed into a key technology. Such requirement for reliable personal identification in computerized access control has resulted in the increased interest in biometrics. ${ }^{5}$ Fingerprints are one of the most mature biometric technologies and are considered legitimate proofs of evidence in courts of law all over the world. Based on the varieties of the information available from the fingerprint we are able to process its identity along with gender, age and ethnicity Fingerprints have some important characteristics that make them invaluable evidence in crime scene investigations:

- An individual's fingerprint ridges are formed during fetal life before birth and remain unchanged even after death.

- Its nature is an individual characteristic that no two persons have been found to be the same, even identical twins.

- Fingerprint classification systems permit the development of file of systematically classified fingerprints and the ability to retrieve a particular file rapidly.

Comparison technique is used for identification so that two factors must be considered one ridge characteristic and another position ofthose characteristics. In process of identification, the fingerprint examiners must compare systematically so that the type of fingerprint pattern (loop, arch or whorl), the finger type (left or right hand, thumb etc.) and the ridge characteristics. The fingerprintridge density can be used to determine whether the fingerprint belong to male or female origin. Some type of the ridge characteristics may be related to the gender. These will assist the fingerprint examiner to direct their search to a particular gender and the investigating officers would save time in nabbing suspects. Identity is a set of physical characteristics, functional or psychic, normal or pathological that defines an individual. Recently, there has been an increased interest in biometric technologies that is human identification based on one's individual features. A fingerprint is an impression of the friction ridges of all part of the finger. A friction ridge is a raised portion of the epidermis on the digits or on the palmar and plantar skin, consisting of one or more connected ridge units of friction ridge skin. Finger-prints may be deposited in natural secretions from the eccrine glands present in friction ridge skin or they may be made by ink or other contaminants transferred from the peaks of friction skin ridges to a relatively smooth surface. Fingerprint patterns are genotypically determined and remain unchanged from birth till death. ${ }^{6}$ Fingerprints collected at a crime scene can be used to identify suspects, victims and other persons who touched the surface, fingerprint scans can be used to validate electronic registration, cashless catering and library access especially in schools and colleges.

The secretions in the fingerprints contain residues various chemicals and their metabolites which can be detected and used for the forensic purposes. ${ }^{2}$ Due to the immense potential of fingerprints as an effective method of identification an attempt has been made in the present work to analyze their correlation with gender of an individual. This correlation between fingerprint pattern and these parameters may help in using fingerprints as an important aid in sex determination and vice versa, thus, enhancing the authenticity of finger-prints in detection of crime and criminals. Positive identification using fingerprints can be established only if 16 to 20 points of similarity exist in the minutiae. ${ }^{7}$ One of the studies done in South Indian population shows that loops are the most commonly occurring fingerprint pattern while arches are the least common. Males have a higher incidence of whorls and females have a higher incidence of loops. Thus, they concluded that there is an association between distribution of fingerprint patterns and gender and prediction of gender of a person is possible based on his fingerprint pattern. ${ }^{8}$

\section{CONCLUSION}

Fingerprint evidence is undoubtedly the most reliable and acceptable evidence till date in the court of law. Due to the immense potential of fingerprints as an effective method of identification an attempt has been made in the present work to analyze their correlation with gender of an individual. The findings of this study could be concluded that fingerprint ridge density is the indicator to establish the gender. Fingerprint 
ridge characteristics may be used to predict the gender. Finally, this study provides information which is useful for forensic science to assist the fingerprint examiners to direct their search to a particular gender, although those are not the rule for identification but it may be used as one of all trends for fingerprintidentification.

\section{REFERENCES}

1. Fingerprint. Available online at : http://en.wikipedia.org/wiki/ Fingerprint

2. Pillay VV. Textbook of Forensic Medicine and Toxicology. 15th ed. Hyderabad: Paras Medical Publishers, 2009. P. 53-94.

3. Kanchan T, Chattopadhyay S. Distribution of Fingerprint Patterns among Medical Students. Journal of Indian Acad of Forensic Med, 2006; 28(2): 65-68.
4. Jain AK, Chen Y, Demerkus M. Pores and Ridges: HighResolution Fingerprint Matching Using Level 3 Features. IEEE Transaction on Pattern Analysis and Matching Intelligence 29 (January 2007)

5. Maltoni D, Maio D, Jain AK, Prabhakar S. Handbook of fingerprint Recognition. 1st ed. New York: Springer; 2003.

6. Vij K. Textbook of Forensic Medicine and Toxicology. 3rd ed. India: Elsevier, 2005. 89-91.

7. Subrahmanyam BV. In: Modi's Medical Jurisprudence and Toxicology. 22nd ed. India :Butterworths, 1999.p. 71-77.

8. Rastogi P, Pillai KR. A study of fingerprints in relation to gender and blood group. J Indian Acad Forensic Med. 2010 Jan; 32(1):11-14 